\title{
Interactive and Lightweight Mechanisms to Coordinate Interpersonal Privacy in Mediated Communication
}

\author{
Natalia Romero, Laurens Boer, and Panos Markopoulos \\ Eindhoven University of Technology \\ $\{$ n.a.romero, 1.m. boer, p.markopoulos\}@tue.nl
}

\begin{abstract}
In this paper we describe three mechanisms that enable people to coordinate their interaction needs with others in their social network. The proposed designs are based on the Privacy Grounding Model [4] that argues the need for lightweight and interactive coordination mechanisms to support the dynamic and dialectic nature of interpersonal privacy coordination.
\end{abstract}

Keywords: Interpersonal Privacy, Common Ground, Coordination, Computer Mediated Communication, Social Communication.

\section{Introduction}

Systems supporting mediated communication aim to facilitate networked communities to engage in different types of interaction, which could not be easily done otherwise. By displaying relevant information about a social network, such systems can, for example, help users to engage in casual interaction with their community [2] or to elicit feelings of connectedness within long-distance family members [1]. However, users of such systems often struggle with the practicalities of keeping their interactions at a desired level [3].

The Privacy Grounding Model [4] provides a theoretical account of how people coordinate interpersonal privacy, which is suggestive of the need for communication systems to provide lightweight and interactive coordination mechanisms that support the dynamic and dialectic nature of interpersonal privacy coordination in mediated settings. The model highlights the importance to support collaborative practices that help community members establish a common understanding of their interaction needs. On the one hand such practices should help people to intentionally contribute to a shared understanding of one's need for interaction; on the other hand such contributions should require low effort comparing to the main communication practices, and allow for ambiguity of one's availability state if desired. The use of such mechanisms should minimize uncertainty and increase understanding of people's interpersonal privacy needs.

Three mechanisms were implemented to demonstrate how lightweight interactivity could support the coordination of interpersonal privacy while communicating without incurring additional effort or disclosing too detailed information if undesired. The mechanisms were deployed as extensions of an existing groupware application, Community Bar (CB) [2]. The original CB was modified so the system will assign a distinctive colour to each user, and incoming messages in the chat window will be coloured according to the sender's colour. 


\section{Interpersonal Privacy Coordination Mechanisms}

Two graphical and one tangible mechanism were implemented in CB to provide representations of one's intention to interact in the chat medium. The one-click mechanism offers an option to click on a message received from someone in one's 'buddy list', which serves as an equivalent of a notification stating: "I've seen your message". The drag-and-drop mechanism offers a 'palette' with a number of predefined messages (e.g., "Coffee break?", "On the phone"), which the user could select from and drag-and-drop under an existing chat message, representing a light reaction (about one's interaction needs) regarding such a message.

Alternatively, the Cylinder provides a tangible interface that uses coloured LEDs to signal incoming messages and alerts sent by others. An alert in CB is similar to the 'nudge' functionality in MS Messenger, a feature used to get someone's attention.

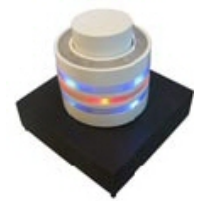

(a)

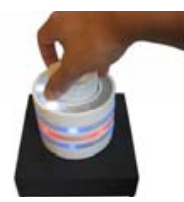

(b)

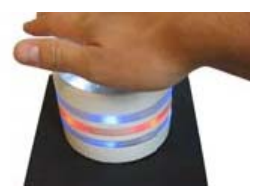

(c)

Fig. 1. The Cylinder: (a) an alert arrives; (b) a time is set; (c) postponing is sent

Once an alert is received, the Cylinder goes up and lights up according to the colour assigned to the particular sender (see Fig 1). The recipient can represent her unavailability in several ways. If she does not react to the movement of the Cylinder, after one minute it automatically gets back to its original position, the light fades away and the sender of the alert receives a text message indicating that there was no reaction to the alert. If the recipient pushes down the tube, a message "I am unavailable" is sent to the sender. Alternatively, the recipient can define a time frame for later contact, and a message "I will be available in about $\mathrm{x}$ to $\mathrm{y}$ minutes" is sent to the sender. The sender can confirm that message by using the one-click mechanism on the received message, which would make the cylinder of the recipient to quickly go up and down. These mechanisms are fully functional and a long-term evaluation is planned to validate their ability to support lightweight privacy coordination.

\section{References}

1. Markopoulos, P., Romero, N., van Baren, J., IJsselsteijn, W., de Ruyter, B., Farschian, B.: Keeping in touch with the family: Home and away with the ASTRA awareness system. In: Proceedings CHI 2004 extended abstracts, pp. 1351-1354 (2004)

2. McEwan, G., Greenberg, S.: Supporting social worlds with the community bar. In: Proceedings of GROUP 2005, pp. 21-30 (2005)

3. Palen, L., Dourish, P.: Unpacking "privacy" for a networked world. In: Proceedings of SIGCHI, pp. 129-136 (2003)

4. Romero, N., Markopoulos, P.: Grounding Interpersonal Privacy in Mediated Settings. In: Proceedings of GROUP 2009 (2009) 